\title{
Unusual fixed drug eruption due to rifampicin
}

\author{
A. K. JAISWAL, S. S. VAISHAMPAYAN, R. VERMA \\ \& K. VARADA \\ Department of Skin, STD and Leprosy, Base Hospital, Lucknow \\ 226002, India
}

Accepted for publication 25 November 1999

\section{Introduction}

Cutaneous reactions due to rifampicin (RFM) are uncommon and amongst these fixed drug eruption (FDE) is especially rare. There have been only three reports of FDE to RFM in the literature so far. ${ }^{1-3}$ In 1985 , Naik et al. first reported a peculiar urticarial type of FDE due to RFM. Here, we describe a second such case with a similar unusual cutaneous drug eruption.

\section{Case report}

A 24-year-old soldier suffering from borderline tuberculoid leprosy (BT) was admitted to the Leprosy Center, Base Hospital, Lucknow for supervised institutional therapy. He was put on cap RFM $600 \mathrm{mg}$ once a month and dapsone $100 \mathrm{mg}$ daily. The patient developed a solitary itchy erythematous urticarial lesion of $3.5 \mathrm{~cm}$ diameter with typical peau d'orange appearance (Figure 1), on the right side of the chest wall about $1 \mathrm{~h}$ after the second monthly dose of RFM. The lesion subsided on its own within $1 \mathrm{~h}$ without any residual hyperpigmentation. A similar lesion at the same site was observed following the third monthly dose of RFM which also subsided within $1 \mathrm{~h}$ without treatment. At this stage, a clinical diagnosis of FDE was made and all drugs were discontinued. Two days later, the patient was subjected to a provocation test with $600 \mathrm{mg}$ of RFM, following which he developed an urticarial lesion at the same site. The provocation test to dapsone did not evoke any cutaneous reaction. Skin biopsy of the lesion revealed slight dermal oedema and sparse eosinophilic infiltration. Interestingly, this cutaneous response to RFM ceased after the fifth dose of RFM.

In our patient, recurrence of a wheal at exactly the same site following administration of RFM is highly suggestive of FDE. Though FDE can be urticarial, the residual hyperpigmentation that follows a classical FDE was not seen. Moreover, the histopathology was

Correspondence to: R. Verma 


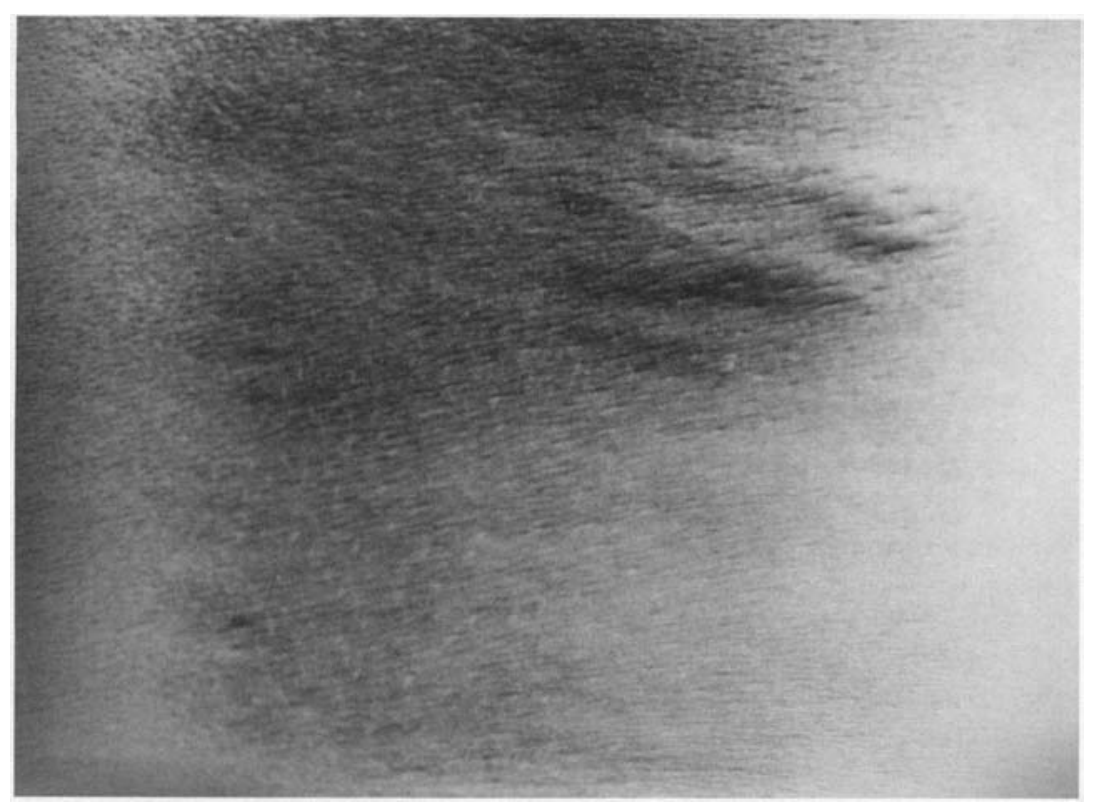

Figure 1. Urticarial lesion on right side of chest.

also consistent with urticaria without pigmentory incontinence. The possibility of a type I lepra reaction was not considered, in view of the onset, further evolution of cutaneous eruption and no change in the pre-existing BT lesions.

The outstanding feature of this case is the unusual non-pigmenting FDE to RFM. Moreover, in contrast to the case reported by Naik et al., the urticarial FDE in our case ceased after the fifth dose of RFM. This is probably because sometimes the inducing drug can be readministered without exacerbation and there may be a refractory period after the occurrence of FDE.

To conclude, this case report raises the question whether the urticarial type of FDE due to RFM is truly rare, or is simply under-reported because of its trivial and transient nature. Further studies on this subject may provide an answer.

\section{References}

1 Naik RPC, Balachandran C, Ramnarayan K. Fixed drug eruption due to rifampicin. Ind J Le pr, 1985; 57: 648-650.

2 Pavithran K. Fixed drug eruption to rifampicin. Ind J Lepr, 1993; 65: 339-341.

3 Stephen SJ. Fixed drug eruption due to rifampicin. Lepr Rev, 1998; 69: 397-399. 